\title{
Shelf Life/Expiration Date Exceeded
}

National Cancer Institute

\section{Source}

National Cancer Institute. Shelf Life/Expiration Date Exceeded. NCI Thesaurus. Code C92106.

A device that has exceeded the period of time/date recommended by the manufacturer for storing the device without a degradation in quality. 